\title{
Immune Checkpoint Functional Assay
}

National Cancer Institute

\section{Source}

National Cancer Institute. Immune Checkpoint Functional Assay. NCI Thesaurus. Code C132165.

Various bioassays designed to measure levels of key regulatory molecules, such as PD-1 (PDCD1), CT LA-4, GITR (TNFRSF18), 4-1BB (TNFRSF9), OX40 (TNFRSF4), and TIGIT, that mediate immune checkpoint receptor pathways or assays to measure proliferation, expression of cell surface activation markers, and cytokine expression induced by these pathways. 